\title{
Labyrinthe
}

$7 \mid 2000$

Numéro 7

\section{Vie d'un groupe statuaire julio-claudien à Mediolanum Santonum}

\section{Emmanuelle Rosso}

\section{(2) OpenEdition}

12 Journals

Édition électronique

URL : http://journals.openedition.org/labyrinthe/805

DOI : 10.4000/labyrinthe.805

ISSN : 1950-6031

Éditeur

Hermann

\section{Édition imprimée}

Date de publication : 30 octobre 2000

Pagination : 103-122

\section{Référence électronique}

Emmanuelle Rosso, «Vie d'un groupe statuaire julio-claudien à Mediolanum Santonum », Labyrinthe [En ligne], 7 | 2000, mis en ligne le 05 mai 2005, consulté le 02 mai 2019. URL : http://

journals.openedition.org/labyrinthe/805; DOI : 10.4000/labyrinthe.805

Ce document a été généré automatiquement le 2 mai 2019.

Propriété intellectuelle 


\title{
Vie d'un groupe statuaire julio-claudien à Mediolanum Santonum
}

\author{
Emmanuelle Rosso
}

1 L'élaboration, au début du Principat, d'un nouveau langage visuel symbolique allant de pair avec une réorganisation de l'espace public urbain par le nouveau régime est un phénomène bien étudié aujourd'hui, qui apparaît comme l'une de ses caractéristiques essentielles, au même titre que la mise en place progressive de pratiques propres à un régime dynastique. Les cycles de portraits impériaux, qui comptent parmi les principales manifestations de ce nouveau langage, illustrent de manière exemplaire ce double aspect du Principat : ils permettent d'affirmer la présence de la domus impériale dans la ville tout en mettant en lumière, par leur composition, les évolutions de la politique dynastique ${ }^{1}$. Dans les provinces, ces cycles iconographiques relèvent sans doute de l'« art officiel», c'est-à-dire d'une production artistique dont les motifs sont choisis à Rome pour être les supports d'une définition du régime et d'une véritable « mise en scène » du pouvoir, mais entrent également dans le cadre d'évergésies dont les auteurs sont les élites municipales ; ils permettent donc d'évaluer la façon dont celles-ci interprètent et expriment l'idéologie impériale et à ce titre ils ont fait l'objet, ces dernières années, de plusieurs travaux : F. Hurlet a étudié grâce à eux le fonctionnement de la "co-régence » exercée par plusieurs princes sous Auguste et Tibère ${ }^{2}$ et $C$. B. Rose a procédé à un recensement systématique de ces ensembles afin de mesurer le rôle de chaque membre de la famille impériale $^{3}$; plusieurs études régionales, portant essentiellement sur l'Italie, ont également été publiées ${ }^{4}$.

Or, les provinces gauloises ont également livré un nombre important de groupes impériaux : outre le cycle statuaire de Béziers ${ }^{5}$, des ensembles julio-claudiens ont été exhumés à Vienne ${ }^{6}$, Avignon ${ }^{7}$, Saint-Bertrand-de-Comminges, Ruscino et Saintes, qui a la particularité d'être le seul ensemble connu à ce jour découvert dans une ville des Trois Gaules, et non en Narbonnaise.

3 Mediolanum Santonum, création ex nihilo née de la conquête romaine ${ }^{8}$, se voit en quelques années promue au rang de capitale de la nouvelle province d'Aquitaine. La fondation de 
cette ville, née en même temps que le Principat et conçue pour être le miroir de Rome, coïncida avec son apogée : dès l'époque augustéenne, la ville prit un visage résolument romain en se dotant d'un centre monumental richement décoré. Cet ensemble urbain ne nous est connu qu'à travers les vestiges remployés dans le rempart tardo-impérial, mais l'abondance des témoignages relevant d'un art impérial officiel permet de comprendre quel fut l'impact du nouveau régime sur l'image urbaine; ils y sont particulièrement éclairants, tant par leur qualité que par leur cohérence : en effet, ils datent tous de la période julio-claudienne, ce qui témoigne d'une présence forte de la première dynastie d'empereurs ${ }^{9}$ et illustre parfaitement la phase de mise en place du régime. D'autre part, ils permettent d'aborder toutes les questions soulevées par ce type de monuments, puisque sont conservés à la fois des bases de statues - de Drusus III (fig. 6) et de Claude, des dédicaces impériales - celles de l'arc routier (fig. 5), qui mentionnent Tibère, Drusus II et Germanicus, des fragments d'effigies en marbre ou en bronze, mais aussi des têtesportraits : une effigie de l'empereur Auguste divinisé (fig. 1), une tête féminine identifiée jusqu'à présent comme une effigie d'Antonia Minor, dont l'étude typologique montre qu'elle représente en réalité Livilla, la sœur de Caligula (fig. 2), une statue masculine acéphale, que l'on peut rapprocher de l'une des bases conservées (fig. 3), et enfin un fragment de statue équestre monumentale en bronze doré (fig. 4). L'importance du dossier saintais tient avant tout au fait que les témoignages sont très rapprochés dans le temps - ils s'échelonnent sur une trentaine d'années, entre le règne de Tibère et celui de Claude - et que la mention des dédicants révèle une étonnante continuité dans la pratique des hommages rendus aux empereurs : plusieurs de ces monuments ont en effet été « financés » par une même famille de notables locaux.

4 La présente étude aura donc pour objet les hommages rendus aux membres de la domus julio-claudienne dans la capitale de l'Aquitaine nouvellement créée ; il s'agira avant tout de retracer la «vie» d'un groupe statuaire en réinsérant les œuvres dans un contexte historique par une comparaison avec d'autres ensembles romains et provinciaux et en mettant en évidence les différentes phases de sa constitution. À l'échelle de la ville, on tentera de les replacer dans leur cadre urbain, au sein de ce qui constitue, on le verra, un véritable « programme » iconographique de grande ampleur pris en charge par des uiri municipales. 


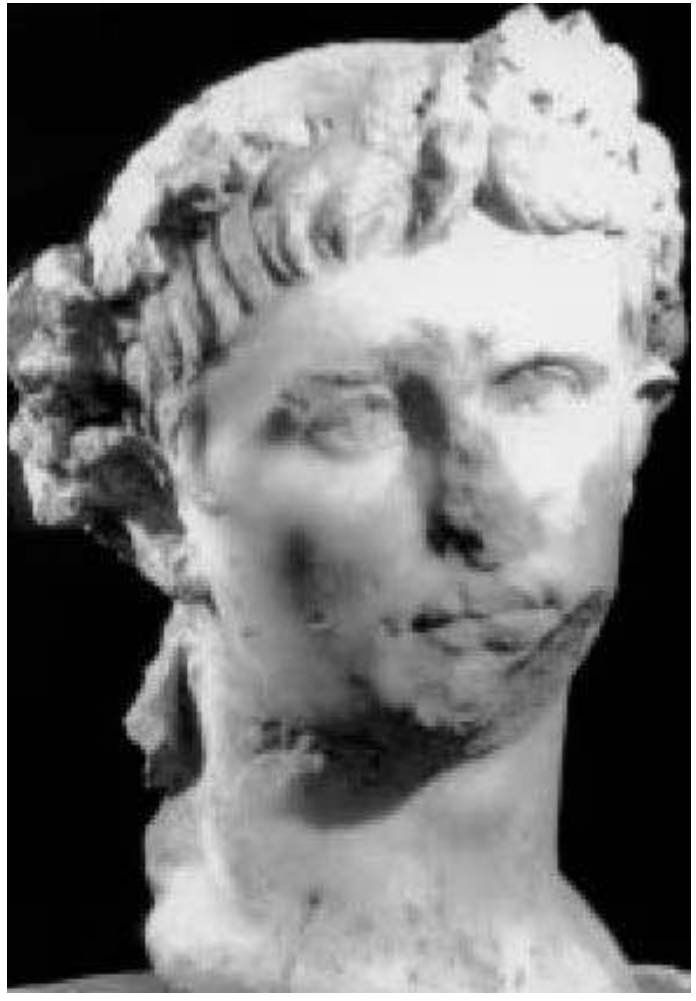

Fig. 1 - Portrait du Divus Augustus. Saintes, Musée Archéologique (Cliché Musée archéologique de Saintes).

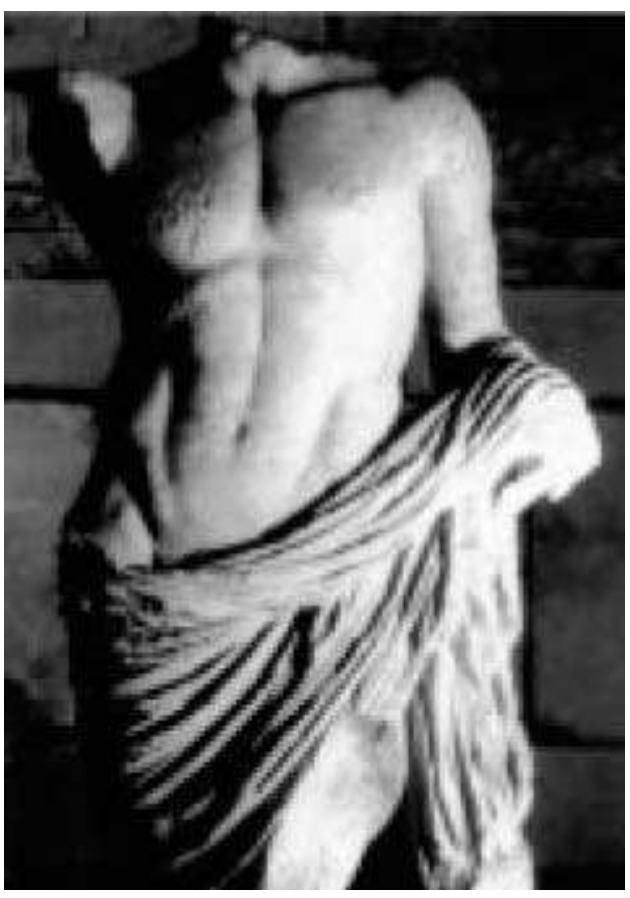

Fig. 3 - Statue acéphale de prince julio-claudien. Saintes, Musée Archéologique (Cliché Musée archéologique de Saintes). 


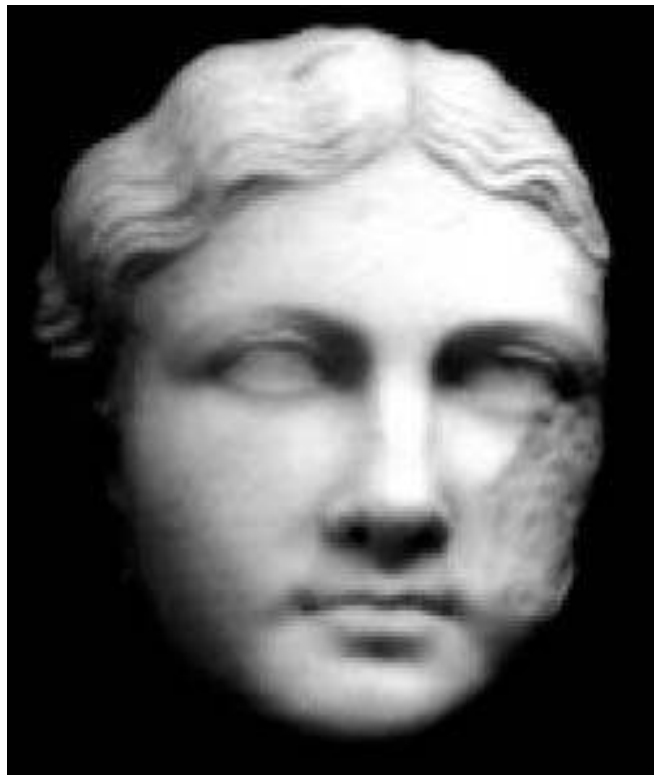

Fig. 2 - Portrait de Livilla. Saintes, Musée Archéologique (Cliché Musée archéologique de Saintes).

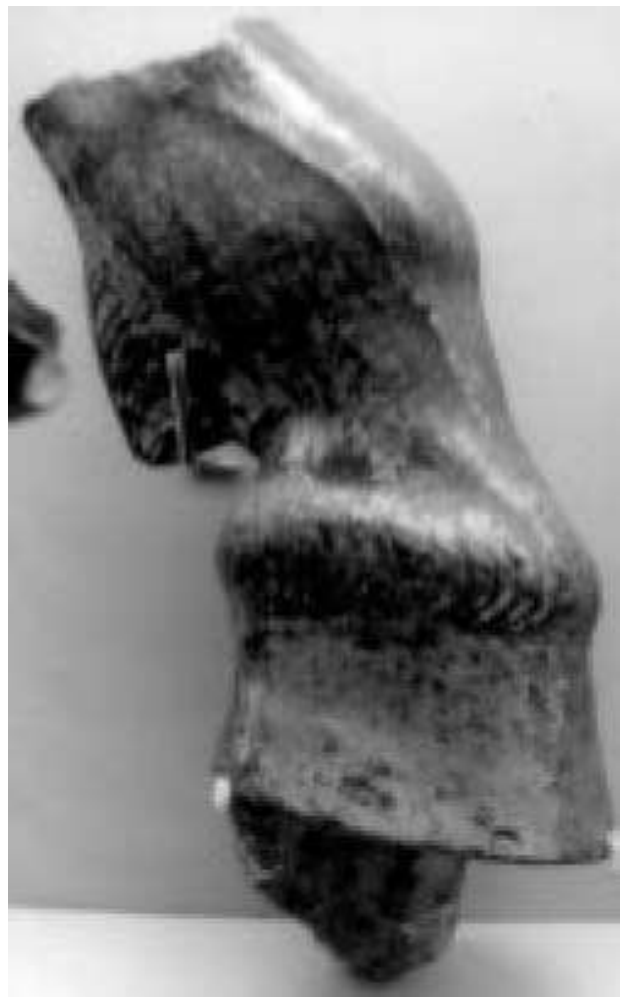

Fig. 4 - Fragment de statue équestre en bronze doré, provenant des environs de l'arc de Saintes. Saint-Germain-en-Laye, Musée des Antiquités Nationales, inv. n 12565 (Cliché E. Rosso). 

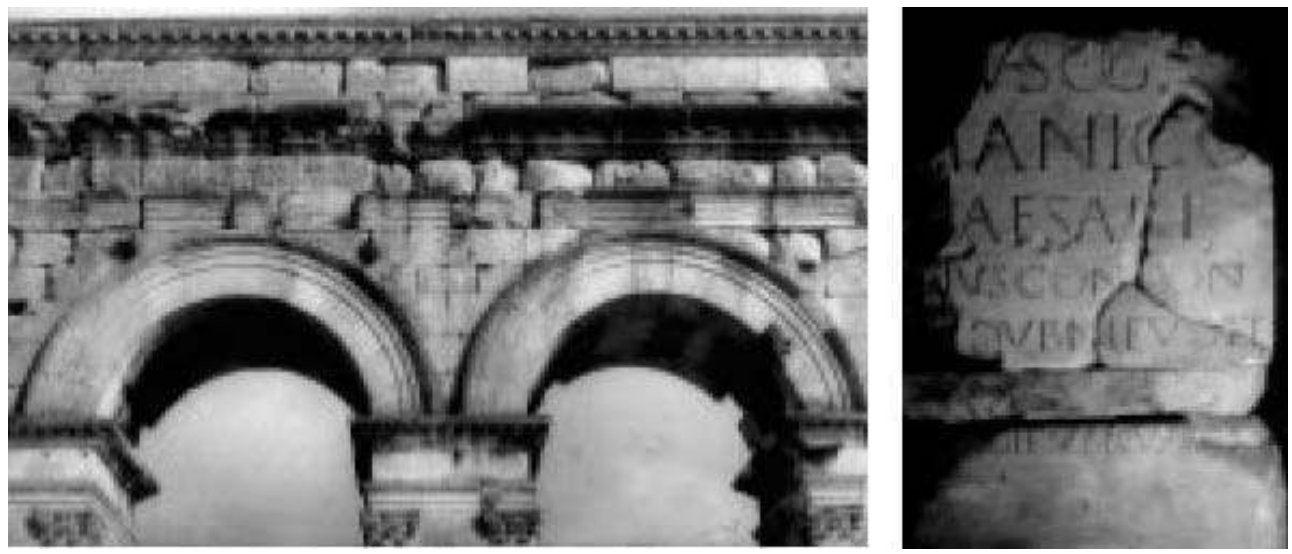

Fig. 5 - L'arc de Saintes : les inscriptions impériales (Cliché Musée Fig. 6 - Base de statue de archéologique de Saintes). Drusus III. Saintes, Musée archéologique (Cliché Musée archéologique de Saintes). 

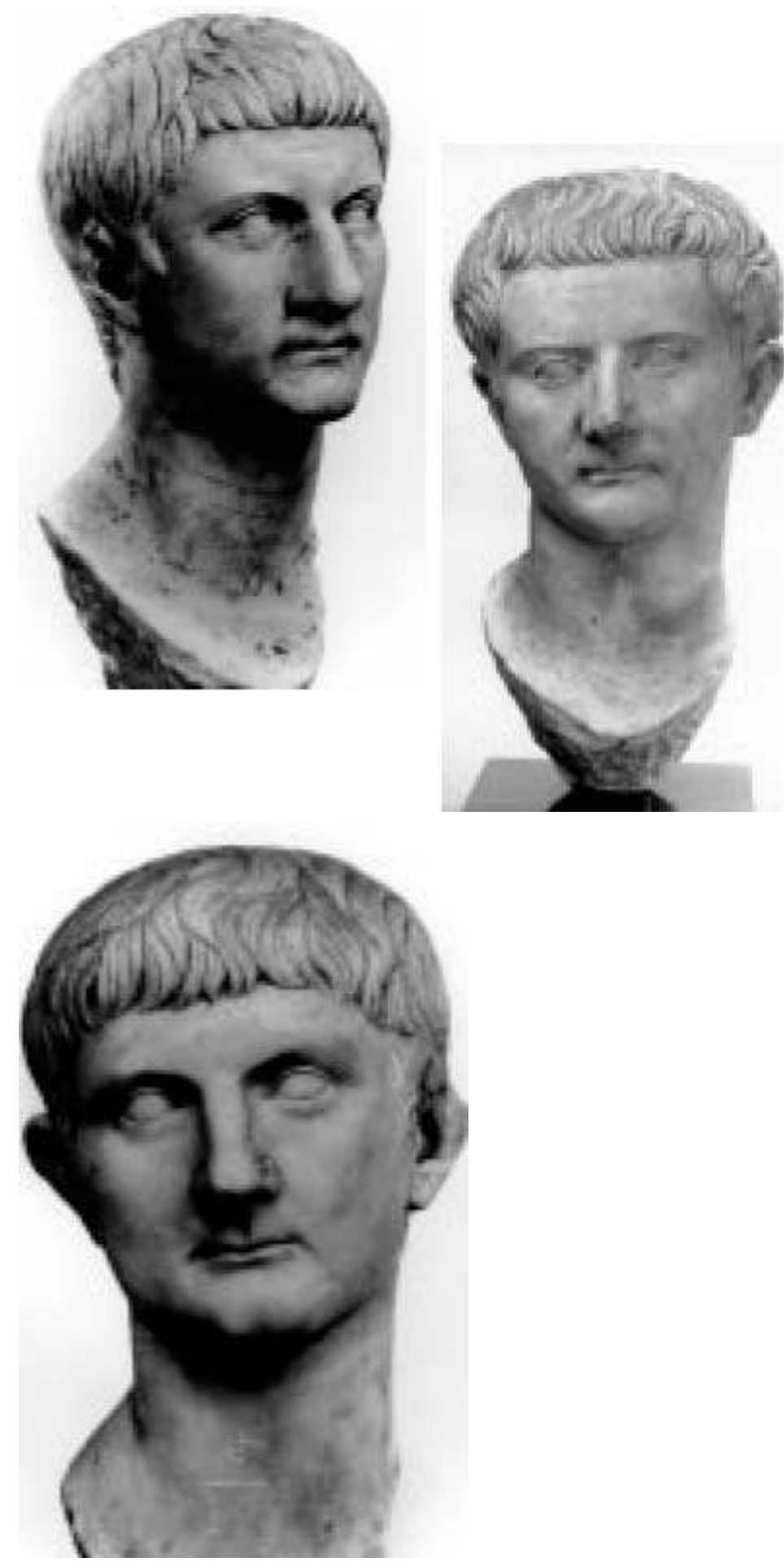

Fig. 7 - Portraits de Drusus le Jeune, Tibère et Germanicus provenant de Béziers. Toulouse, Musée Saint-Raymond, inv. n 30 011, 30009 et 30010 (Cliché CNRS Centre Camille Jullian).

5 Un premier ensemble statuaire, constitué des effigies de Tibère, Drusus II et Germanicus, couronnait le seul monument en grand appareil encore debout à Saintes, l'arc routier à deux baies destiné à marquer l'entrée dans la ville et le passage du fleuve ; ce groupe a entièrement disparu. Sont conservées toutefois les trois dédicaces qui figurent à l'attique du monument, sur la façade occidentale - donc du côté de la ville (fig. 5). La tripartition de l'inscription, qui décrit des champs épigraphiques juxtaposés, correspond sans doute à 
la disposition des effigies qui surmontaient la plate-forme supérieure ${ }^{10}$ de l'arc : la titulature de Tibère occupe le centre de la composition et s'étend sur deux lignes, contrairement à celles des princes qui sont plus étroites et en comportent trois. Cette position de la statue de Tibère semble le désigner comme destinataire privilégié du monument. Il était entouré de son fils Drusus et de son neveu et fils adoptif Germanicus ; l'édifice ne doit donc son nom d'«arc de Germanicus» qu'au meilleur état de conservation de la dédicace qui le mentionne. Quelles sortes de représentations pouvaient couronner cet édifice, et quel est donc l'aspect probable de ce groupe statuaire ?

6 À Rome même, mais aussi dans les provinces, nombreux sont les arcs honorifiques qui, dès le règne d'Auguste, comportent des groupes de statues-portraits ${ }^{11}$. Le groupe tripartite y est une formule répandue - il ne constitue d'ailleurs pas une spécificité de l'iconographie des arcs, mais se rencontre de façon récurrente dans toutes sortes de monuments à signification dynastique : l'empereur régnant y apparaît avec ses enfants ou petits-enfants, parfois l'un de ses parents, dans des compositions destinées à exhiber les liens familiaux, dans un but de présentation des successeurs potentiels et donc de légitimation du pouvoir ${ }^{12}$; sous le règne de Tibère, Germanicus et Drusus apparaissent très régulièrement aux côtés de l'empereur dans des groupes qui nous sont connus par des séries de dédicaces ou de portraits, à Rome ${ }^{13}$ bien sûr, mais aussi à Éphèse, Clazomènes, Lindos, Palmyre, ou encore Sagonte. L'iconographie de l'arc de Saintes n'a donc rien d'inattendu dans sa composition et correspond en outre, on le verra, à une phase de la politique de succession de Tibère particulièrement bien documentée, tant à Rome qu'en Gaule ${ }^{14}$.

7 En ce qui concerne le type statuaire des effigies de ces princes, nous avons la chance de posséder un témoignage d'importance, malgré le caractère extrêmement fragmentaire de sa conservation : il s'agit d'un sabot de cheval en bronze, qui a été découvert en 1870 à proximité de l'arc ${ }^{15}$ (fig. 4). Ce fragment, si infime soit-il, nous apprend que l'arc était surmonté d'un groupe en bronze doré comprenant au moins une statue équestre grandeur nature ; il est bien évidemment très tentant de penser que c'est Tibère luimême que cette statua equestris inaurata - pour reprendre l'expression de l'arc de Caius César, à Pise ${ }^{16}$ - figurait, puisque l'empereur occupait sur l'arc une position centrale et spatialement plus importante. On pourrait certes songer aussi à un char triomphal, mais L. Maurin fait remarquer à juste titre que "l'étroitesse de la plate-forme supérieure rend difficile de restituer au centre la statue de l'empereur dans le char traîné par un quadrige $»^{17}$; on imagine donc plutôt un groupe constitué d'une statue équestre de l'empereur régnant flanquée des deux effigies en pied - cuirassées (triumphali ornatu) ou, moins probablement, en toge - de Germanicus et Drusus II. Si la plate-forme supérieure est étroite, elle est en revanche assez longue - plus de quinze mètres, de sorte qu'on peut penser que la statue équestre se présentait de profil, l'empereur regardant du côté de la ville.

8 La présence de cette statue équestre confère à l'ensemble une connotation militaire, voire triomphale, évidente ; la nature des représentations qui couronnaient l'arc semble donc fournir un premier indice susceptible d'éclairer la signification du monument, au plan idéologique ; mais c'est surtout sa datation exacte qui permettrait de l'évaluer au plus près, en le situant précisément dans le contexte politico-dynastique tourmenté de la décennie qui suivit l'accession au trône de Tibère. Malheureusement, cette datation fait difficulté. 
Il est communément admis que l'arc fut dédié à l'occasion de la charge que le dédicant, $C$. Iulius Rufus, a exercée à l'autel du Confluent, à Lyon ${ }^{18}$, en tant que prêtre du culte impérial ; on a également proposé d'y voir une réalisation commémorant l'achèvement de la route d'Agrippa ${ }^{19}$. L'origine et la raison d'être de cette initiative seraient donc purement régionales. Toutefois, s'il est vrai que l'arc de Saintes est avant tout un arc routier ayant fonction de repère, de «signal » à un point focal de la ville, la présence de statues impériales dans sa partie supérieure en fait d'emblée un édifice honorifique et commémoratif ; de toute évidence, la nature même des dédicataires en infléchit considérablement la signification politique ; en effet, même si le dédicant fait figurer son nom en bonne place - l'inscription de Rufus court sur toute la largeur de l'arc et est répétée sur les deux faces ${ }^{20}-$ lui-même n'a sans doute jamais été représenté aux côtés des membres de la famille impériale, alors que la présence d'effigies de personnages privés sur ce genre d'édifices est un fait attesté par ailleurs ${ }^{21}$. Dans la mesure où seuls sont figurés, à Saintes, des princes de la Domus Augusta, l'histoire régionale semble s'effacer devant celle du « centre du pouvoir» : dans cette perspective, l'arc de Saintes doit être compris comme un monument honorifique à tonalité dynastique qui, en même temps qu'il réaffirmait la légitimité des «co-régents» Germanicus et Drusus, exaltait leurs succès - militaires entre autres. Ainsi, du fait du caractère officiel de ce monument élevé à la gloire de la famille impériale, il est fort probable que la charge exercée par le dédicant n'ait été que le prétexte et non véritablement l'occasion qui a présidé à son érection. En effet, si le mauvais état de conservation des titulatures impériales n'autorise pas une datation extrêmement précise, il est en revanche certain que la dédicace est intervenue à un moment-clé du principat de Tibère.

Les titulatures de Tibère et de Drusus le Jeune ${ }^{22}$ sont extrêmement mutilées et ne fournissent aucun indice chronologique éclairant ; c'est donc celle de Germanicus qui permet de dater cet $\operatorname{arc}^{23}$, qui a été dédié après le premier janvier de l'année 18 apr. J.-C. ; toutefois, cette titulature correspond également à celle qui était la sienne au moment de la mort du prince, le 10 octobre 19 apr. J.-C., de sorte qu'il est, en toute rigueur, impossible de savoir à partir des seuls indices épigraphiques si la dédicace de l'arc est intervenue avant ou après son décès.

11 Les deux possibilités sont envisageables. P. Grimal excluait totalement la seconde ${ }^{24}$, en alléguant que, dans ce cas, la dédicace aurait nécessairement mentionné le décès de Germanicus, puisqu'il était selon lui impossible de faire figurer sur un même monument un personnage décédé et des vivants ${ }^{25}$. Depuis lors, la documentation sur les hommages officiels rendus aux princes défunts (et plus particulièrement à Germanicus) s'est considérablement accrue, de sorte que l'on pourrait être tenté de mettre cette dédicace en rapport avec les honneurs posthumes qui lui furent décernés : le témoignage le plus important en est le senatus consultum de supremis honoribus Germanici, dont la célèbre Tabula Siarensis ${ }^{26}$, découverte en 1982 près de Séville, nous a conservé le texte : il décrétait l'érection, à Rome, in Circo Flaminio, d'un ianus surmonté d'une statue de Germanicus César in curru triumphali, c'est-à-dire sur un char triomphal, accompagnée d'effigies des membres de la famille du prince ${ }^{27}$. Cette décision du Sénat romain fut sans doute suivie de réalisations comparables dans les provinces de l'Empire - outre les arcs du Mont Amanus, en Syrie, et de Mayence ${ }^{28}$ - ce que mentionne du reste le texte même du décret, qui envisage la possibilité d'autres dédicaces « en tout autre lieu que (...) Tibère César Auguste jugerait plus adapté, dans les régions dont il avait confié l'administration et la protection à Germanicus César sur l'avis du Sénat ${ }^{29}$. Comme la Gaule fait précisément 
partie de ces régions ${ }^{30}$, l'arc situé à Saintes, capitale de province, pourrait fort bien s'insérer dans la série des monuments provinciaux inspirés par ce contexte d'honneurs funéraires rendus à Germanicus ${ }^{31}$. Si tel est le cas, le monument constitue, au moins en partie, un hommage à caractère funéraire, " ad conservandam memoriam Germanici Caesaris, qui mori numquam debuit », selon l'expression même du décret ${ }^{32}$; la présence de Tibère à ses côtés correspondrait alors à un respect du «protocole $\|^{33}$. Quant à Drusus, il constituait une promesse d'avenir, puisqu'il était appelé à prendre la relève de son cousin dans le rôle de co-régent. De fait, le cas d'un monument destiné à honorer en priorité les héritiers présomptifs tout en réservant une place privilégiée à la personne de l'empereur ne serait pas sans précédent en Gaule, si l'on suit l'hypothèse que Pierre Gros a formulée à propos de l'arc d'Arausio (Orange) ${ }^{34}$ : ce monument, prévu pour être l'hommage des Secundani à leur général Germanicus, aurait en effet été « rendu à Tibère » à la suite de réticences de l'empereur envers les honneurs considérables dont son fils adoptif faisait l'objet. Toutes proportions gardées, l'arc de Saintes aurait une signification comparable à celle de l'arc d'Arausio, qui lui est postérieur de quelques années ${ }^{35}$.

Le parallèle avec les hommages rendus au prince défunt et plus particulièrement avec l'arc du Circus Flaminius présente toutefois certaines limites : outre le fait qu'à Rome ne sont présents ni Tibère ni Drusus - signe qu'il s'agissait avant tout d'un monument à

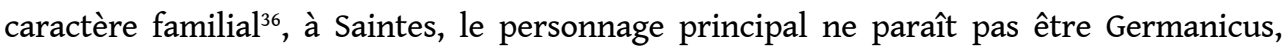
mais l'empereur lui-même. Ces deux arguments semblent plaider en faveur d'une datation antérieure à la mort de Germanicus ; c'est l'hypothèse de P. Grimal ${ }^{37}$, qui a mis en rapport le monument de Saintes avec la série des arcs élevés en l'honneur de Germanicus et de Drusus II dans le courant de l'année 19, dont celui du Forum d'Auguste a dû fournir le paradigme. De fait, bien plus nombreux encore sont les groupes statuaires tripartites qui ont honoré l'empereur et ses deux successeurs potentiels avant cette date, à partir de 14 apr. J.-C. : il s'agissait alors d'établir un parallélisme avec le groupe que formaient Auguste et les princes de la jeunesse, devenus les fils de l'empereur. La situation de Germanicus et de Drusus II au sein de la domus impériale était similaire, puisqu'ils étaient, eux aussi, à peu près du même âge, et avaient effectué des carrières parallèles. Au sein de cette période de six ans, le triomphe de Germanicus De Germanis, intervenu en 17, accéléra sans aucun doute le rythme des dédicaces; sur le plan symbolique, la symétrie avec les fils d'Agrippa n'en devient que plus claire : il s'agit en effet de signifier que le pouvoir est désormais aux mains de la branche claudienne de la gens - qui ne le cède en rien à la lignée du gendre d'Auguste, comme l'atteste la brillante carrière des fils de Tibère.

13 Par conséquent, si la dédicace de l'arc de Saintes est à situer au cours de l'année précédant la mort de Germanicus, l'ensemble statuaire était probablement destiné à exalter une période de stabilité du pouvoir et de grands espoirs pour l'avenir de la dynastie, dont la prospérité semblait garantie par les succès des successeurs désignés : en Gaule, les trois portraits du groupe de Béziers figurant l'empereur et ses fils ${ }^{38}$ illustrent parfaitement cette phase - ils sont en effet datables des années 14 à 19 apr. J.-C. - et revêtent donc une signification similaire, sur le plan politique ${ }^{39}$; il faut donc sans doute imaginer les têtes-portraits des statues de Saintes figurées selon les mêmes types iconographiques qu'à Béziers (fig. 7).

14 En l'absence de critère chronologique précis, il est malheureusement impossible de trancher en faveur de l'une ou l'autre datation ${ }^{40}$, car il faut tenir compte également du délai qui a séparé la décision de construire le monument et sa réalisation effective ${ }^{41}$. Que 
l'arc ait été dédié dans le courant de l'année 18 ou après le 10 octobre 19, l'occasion de son érection paraît plus profondément liée à un événement de la vie politique de l'Empire qu'à la carrière personnelle du dédicant, d'autant plus que Tibère, Germanicus et Drusus II comptent parmi les princes de la domus Augusta le plus souvent honorés en Gaule ${ }^{42}$. Qu'il y ait un lien entre la prêtrise à l'autel du Confluent de Rufus et cette dédicace n'est certes pas douteux ; mais ce lien n'est peut-être pas aussi strict qu'on l'a parfois laissé entendre - le seul exercice de cette charge ayant déterminé chronologiquement l'érection de l'arc - et ce dernier n'est sans doute pas autre chose qu'un témoignage "normal», voire obligé - d'allégeance envers la famille impériale de la part d'un riche notable qui constituait à l'échelon local, de par ses fonctions, l'un des représentants les plus autorisés du pouvoir central.

Les dédicaces de l'arc, on l'a vu, permettent de distinguer une première phase d'hommages à Saintes, sous Tibère ; mais nous possédons également des témoignages pour les périodes ultérieures. Une seconde phase est représentée en premier lieu par la base de statue de Drusus César, le second fils de Germanicus, qui fut découverte dans le rempart du Bas-Empire : comme pour les inscriptions de l'arc, la datation de cette dédicace fait problème ; l'ellipse de la titulature que mentionnent les textes honorant le prince de son vivant semble toutefois indiquer qu'il s'agit d'une dédicace posthume datant du règne de Caligula. L'examen de la face supérieure du bloc ne donne aucune indication précise sur le type statuaire de l'effigie qui surmontait le piédestal ; on peut seulement déduire de ses dimensions qu'il s'agissait d'une effigie grandeur nature. Or, on a découvert à proximité du rempart une statue acéphale représentant un jeune homme en semi-nudité héroïque. Dans les premiers temps du Principat, ce type statuaire était réservé aux représentations de divinités ou de personnages impériaux et, avant Claude, à celles des princes défunts. La taille de l'effigie - dont les dimensions correspondent assez bien à celles de la face supérieure du piédestal - son type iconographique, son lieu de découverte, tout semble inviter à rapprocher cette statue de l'inscription dédiée à Drusus III ; si cette hypothèse est exacte, nous serions en présence d'un cas unique dans le corpus gaulois de correspondance entre une base et la statue qui l'accompagnait.

À la même phase d'hommages peut être rattaché également le portrait d'Auguste,, dont la datation posthume s'impose du fait de la présence, autour de la tête, de cavités destinées à recevoir une couronne de rayons, signe de divinisation (fig. 1) ; des critères d'ordre stylistique permettent par ailleurs de préciser qu'il a sans doute été réalisé pendant le règne de Caligula ; la statue de Drusus III et celle d'Auguste seraient donc contemporaines. Mais il est un troisième portrait susceptible d'entrer en ligne de compte pour cette période ; il s'agit d'une tête féminine assez mutilée (fig. 2) dont un réexamen au Musée de Saintes m'a amenée à remarquer la présence, sur le front, d'une "couronne" de petites boucles coquillées en faible relief caractéristique d'une série d'effigies attribuables à Livilla, la sœur de Caligula ; L. Maurin ne s'y était d'ailleurs pas trompé, puisqu'il avait vu dans ce portrait Antonia Minor, sa grand-mère - la ressemblance physique naturelle se double ici d'un évident phénomène d' "Angleichung " parfaitement voulu - et $\mathrm{y}$ avait vu une réplique du règne de Caligula. Nous possédons donc à Saintes une série de trois portraits d'époque caliguléenne. La "fourchette" chronologique très restreinte au sein de laquelle ils ont été réalisés, mais aussi la proximité de leurs lieux de découverte respectifs conduisent à les envisager ensemble, sinon comme un véritable groupe unitaire formé d'éléments parfaitement 
contemporains, tout au moins comme un ensemble cohérent où la signification d'une œuvre s'éclaire par la présence des autres.

La découverte dans une même ville de portraits du divus Augustus, de Drusus César et de Livilla sous le règne de Caligula n'a rien pour surprendre : Auguste est tout à fait normalement présent dans tous les ensembles dynastiques de l'époque julio-claudienne ; à Saintes, comme à Béziers, il trouve également sa place en tant que fondateur de la ville. Livilla a été fréquemment honorée en même temps que Drusilla, la sœur bien-aimée de l'empereur ; enfin, de la même façon, Drusus III, le troisième fils de Germanicus, poursuivi par la haine de Séjan et décédé en 33 apr. J.-C., réapparaît dans les groupes statuaires sous le règne de son frère et reçoit des honneurs posthumes conjointement à Néron César ; il s'agit alors de recréer une unité symbolique autour de la figure de Germanicus.

Dès lors, le parallélisme avec le groupe de l'arc devient particulièrement signifiant : d'un côté, avec ce dernier, nous avons la célébration de l'avènement d'une branche claudienne prospère, l'empereur apparaissant entouré de ses fils dont la carrière militaire et diplomatique est à la fois brillante et rapide ; de l'autre, quelque vingt années après, c'est la nombreuse descendance de Germanicus qui est honorée : Néron et Drusus César, après avoir été les héritiers présomptifs de Tibère à la tête de l'Empire au même titre que Drusus II et leur père Germanicus, apparaissent après leur mort et sous le règne de leur frère comme une paire de "successeurs idéaux » comme ont pu l'être avant eux Caïus et Lucius César, éternels « princes de la jeunesse ».

Une troisième et dernière phase d'hommages est représentée par un témoignage isolé, une base de statue de l'empereur Claude datant de l'année 49 apr. J.-C. ${ }^{43}$; une nouvelle fois, il s'agit d'une inscription provenant du rempart tardo-antique de la ville, ce qui donne à penser que son lieu d'exposition originel était proche de celui des effigies caliguléennes.

domi nobiles

La correspondance entre les effigies de l'arc et celles qui étaient peut-être situées sur le forum ou en un autre lieu public de la ville se comprend d'autant mieux si on examine les dédicants des différentes statues; si l'arc routier a été dédié par C. Iulius Rufus, la statue de Drusus César l'a été par un certain C. Iulius [Co]gidubnus qui est selon toute vraisemblance son cousin ; quant au dédicant de la statue de Claude, ce n'est autre que C. Iulius Victor fils, qui est connu à Saintes pour avoir dédié le mausolée de son père Caïus ${ }^{44}$, dont la généalogie permet de reconstituer le stemma familial jusqu'à l'arrière grand-père de Rufus, Epotsoviridius.

21 On observe donc une remarquable continuité dans la pratique des hommages aux membres de la domus julio-claudienne au sein de cette famille d'origine santonne dont on peut suivre les dédicaces sur au moins deux générations : il semble s'être instauré une véritable tradition familiale chez des personnes visiblement très attachées à l'idée impériale - attachement qui résulte peut-être de l'octroi de la citoyenneté romaine au grand-père de Rufus de la part de César ou d'Auguste, comme le suggère le gentilice Iulius - et il ne serait pas surprenant que les effigies de Livilla et du Divus Augustus aient elles aussi été dédiées par des Iulii. Il ne peut s'agir bien sûr que d'une hypothèse que seule rend probable la cohérence, sur le plan idéologique, du regroupement des personnages représentés. De même, il dut y avoir également des effigies de Caligula et peut-être d'Agrippine l'Ancienne, qui, en tant que fille de Julie, assurait un lien direct avec la Gens Iulia. 

courte période au cours de laquelle elles ont été dédiées - entre 18 et 42 apr. J-C - et on peut parler d'un véritable programme iconographique ${ }^{45}$, réalisé à l'échelle de toute une ville, en raison de cette unité extrême - proximité des lieux de découverte, unité chronologique, unité des dédicants, cohérence idéologique - qui est extrêmement rare dans la mesure où les témoignages sont généralement sporadiques et espacés dans le temps. Si l'on ajoute le fait que $C$. Iulius Rufus fut également le dédicant, sous Tibère, de l'amphithéâtre des Trois Gaules à Lyon ${ }^{46}$, capitale du culte impérial provincial - où il prend soin de rappeler ses origines santonnes, on peut conclure que ce programme monumental dépassait largement le simple cadre urbain pour s'étendre aux dimensions de toute une province : les voyageurs quittant Saintes pour rejoindre Lyon, qui passaient sous l'arc et pouvaient admirer les statues des membres de la famille de Tibère offertes par C. Iulius Rufus ${ }^{47}$, retrouvaient ce même personnage à l'entrée de Lyon lorsqu'ils apercevaient l'amphithéâtre non loin du sanctuaire consacré à Rome et à l'empereur, l' Ara Romae et Augusti ad Confluentem ${ }^{48}$.

Ili de Saintes, "grands notables de petite ville ", selon l'expression de W. Seston ${ }^{49}$ appartiennent par conséquent à une élite municipale qui rend hommage à l'idée impériale et reconnait comme légitime l'ordre imposé par Rome plus qu'elle ne fait preuve de marques d'allégeance spontanées. Leurs actes d'« évergétisme » qui sont peutêtre en relation avec leur entrée ou leur sortie de charge, pouvaient en tout cas constituer, dans certains contextes politiques particuliers, des obligations ; ici, l'autorisation nécessaire de l'empereur pour l'érection d'un monument tel que l'arc pourrait cacher une pression des autorités. Enfin, il est bien évident que ces dédicaces font apparaître le nom de ces personnages en position privilégiée, de sorte qu'elles deviennent, de manière indirecte, les instruments de leur propre promotion ; en effet, la proximité que ces personnages créent avec l'empereur par le biais de tels hommages ne peut que rejaillir positivement sur la perception qu'avaient d'eux les habitants de la ville. Il ne faut donc pas perdre de vue la dimension de propagande personnelle qu'ils comportent $^{50}$; le cas de l'arc de Saintes est à cet égard particulièrement significatif, puisque l'inscription mentionnant les noms et la carrière du donateur est la seule qui apparaisse sur les deux faces, les dédicaces impériales n'étant gravées que du côté de la ville; le personnage principal de cette mise en scène est donc en quelque sorte $C$. Iulius Rufus lui-même.

Il est intéressant de remarquer que l'origine sociale des dédicants telle qu'elle est repérable à travers l'exemple de Saintes est donc parfaitement semblable à celle que l'on trouve dans les autres cités gauloises mais aussi dans toutes les autres provinces de l'Empire romain ; F. Hurlet fait des constatations identiques pour les règnes d'Auguste et de Tibère, en remarquant qu'en dehors des communautés constituées, les individus privés finançant la réalisation des hommages statuaires «ont en commun d'appartenir aux cercles les plus proches du pouvoir $»^{51}$.

On peut donc restituer trois " phases » d'hommages statuaires à la domus julio-claudienne à Saintes : tout d'abord, une phase tibérienne qui comprend la dédicace de l'arc; en second lieu, une phase caliguléenne pendant laquelle furent érigées les effigies d'Auguste, de Livilla et de Drusus III ; enfin, un enrichissement à l'époque claudienne, avec la dédicace d'une statue de Claude.

Labyrinthe, 7 | 2005 


\begin{tabular}{|l|l|l|}
\hline Date, règne & Personnages honorés & Personnages honorés \\
\hline \hline & dans l'épigraphie & dans l'iconographie \\
\hline \hline Tibère & - Tibère & Statue équestre de Tibère \\
\hline \hline (19 apr. J.-C.) & - Drusus II & (?) \\
\hline \hline & - Germanicus & Statuae pedestres des \\
\hline \hline Caligula & (dédicant : C. Iulius Rufus) & deux princes (?) \\
\hline \hline Caligula & - Drusus III & Prince héroïsé \\
\hline \hline (37-42 apr. J.-C.) & (dédicant :C. Iulius & (Drusus III ?) \\
\hline \hline Claude & [Co]gidubnus) & \\
\hline \hline (48-49 apr. J.-C.) & (dédicant : C. Iulius Victor II) & \\
\hline \hline (vers 37-39 apr. J.-C.) & \\
\hline \hline
\end{tabular}

$\mathrm{Au}$ cours des quatre premières décennies de notre ère, deux générations de Iulii semblent donc avoir orchestré de façon presque exclusive, dans leur patrie d'origine devenue capitale de province, la constitution et l'enrichissement de l'ornatus civique, dont les statues impériales constituent l'élément le plus significatif ; en ce sens, ils ont modelé de façon durable l'image urbaine en imposant à la fois leur présence et celle du nouveau régime, au sein d'un jeu de relations - et de relais - complexe où se mêlent les impératifs apparemment contradictoires mais finalement convergents d'autoreprésentation (donc de propagande personnelle) et de glorification du pouvoir, dans un mouvement d'influences réciproques ${ }^{52}$. L'exemple de Saintes illustre donc de manière éclatante à quel point il faut tenir compte, dans l'analyse des monuments relevant de «l'art du prince » - et Paul Zanker insistait récemment sur ce point ${ }^{53}$, des intérêts particuliers que poursuivaient les différents commanditaires et exécutants intervenant, à divers niveaux, dans leur élaboration ; cette multiplication des protagonistes peut être déroutante en ce qu'elle empêche d'évaluer la part des initiatives locales et celle des prescriptions venues de Rome ; elle invite en tout cas à tenter de comprendre ces œuvres selon un point de vue double. Ainsi, de Saintes à Lyon, d'une capitale des Gaules à l'autre, c'est bien un même programme d'exaltation parallèle de la famille impériale et de celle de 
ses représentants locaux qui est à l'œuvre, par le biais du dialogue qui s'instaure entre le Prince et ses sujets.

\section{BIBLIOGRAPHIE}

Dans les notes de bas de page, les références bibliographiques ont été abrégées par le nom de l'auteur suivi de la date de publication de l'article ou de l'ouvrage. Les abréviations des titres de revues sont celles de l'Archäologische Bibliographie.

BEDON R., Les Villes des Trois Gaules de César à Néron, dans leur contexte historique, territorial et politique, Paris, 1999.

Boschung D., Die Bildnisse des Augustus (Das römische Herrscherbild, I-2), Berlin, 1992. -, « Die Bildnistypen der iulisch-claudischen Kaiserfamilie : ein kritischer Forschungsbericht ", JRA, 6,1993, p. 38-79.

ESPÉRANDIEU E., Épigraphie romaine du Poitou et de la Saintonge, Paris-Melle, 1889.

_, Recueil général des bas-reliefs, statues et bustes de la Gaule romaine, Paris, 1908-1951.

HURLET F., Les Collègues du prince au temps d'Auguste et de Tibère : de la légalité républicaine à la légitimité dynastique, (Collection de l'École Française de Rome, 227), Rome, 1996.

KIENAST D., Römische Kaisertabelle : Grundzüge einer römischen Kaiserikonographie, Darmstadt, 1990.

MAURIN L., Saintes antique des origines à la fin du vI siècle apr. J.-C., Saintes, 1978.

-, Inscriptions latines d'Aquitaine, Santons, Bordeaux, 1994.

NIEMEYER H.-G., Studien zur statuarischen Darstellung der römischen Kaiser, Berlin, 1968.

RoSE C. B., Dynastic commemoration and imperial Portraiture in the julio-claudian Period, Cambridge, 1997.

WUILLEUMIER P., Inscriptions latines des Trois Gaules, Paris, 1963.

ZANKER P., Augustus und die Macht der Bilder, Munich, 1987.

\section{NOTES}

*. Ce travail fait suite à un mémoire de DEA intitulé : « Portraits impériaux de Narbonnaise, d'Aquitaine et de Lyonnaise : statuaire et épigraphie », soutenu en juin 1999 à l'Université de Paris IV, sous la direction de M. le Professeur J.-Ch. Balty, que je tiens à remercier tout particulièrement pour ses conseils, suggestions et corrections. Que soient également remerciés M. Catherine Duffault, G. Dumerchez (Conservation du Musée archéologique de Saintes) ainsi que M. D. Cazes, conservateur du Musée Saint-Raymond de Toulouse, qui m’ont autorisée à évoquer ces œuvres et à en publier des clichés. 
1. J.-Ch. Balty, « Groupes statuaires impériaux et privés de l'époque julio-claudienne », Ritratto ufficiale e ritratto privato (Quaderni de La Ricerca scientifica, 116), Rome, 1988, p. 31-45.

2. F. Hurlet, Les Collègues du prince au temps d'Auguste et de Tibère : de la légalité républicaine à la légitimité dynastique, coll. EFR, 227, Rome, 1996.

3. C. B. Rose, Dynastic commemoration and imperial portraiture in the julio-claudian period, Cambridge, 1997.

4. C. Saletti, «I cicli statuari giulio-claudi della Cisalpina. Presenze, ipotesi, suggestioni », Athenaeum, 81, 1993, p. 365-390 ; I. Cogitore, « Séries de dédicaces italiennes à la dynastie julioclaudienne », MEFRA, 104, 1992-2, p. 817-870.

5. J.-Ch. Balty, D. Cazes, Portraits impériaux de Béziers. Le groupe statuaire du forum, Toulouse, 1995.

6. F. Salviat, « Portraits d'Auguste à Vienne, de Tibère au Musée de Lyon : un relief "dynastique" en Gaule ? », RAN, 16, 1983, p. 135-144.

7. F. Salviat, « Tibère, Drusus le Jeune et le "forum" d'Avignon », RA, 1979, 2, p. 259-268 ; J.-Ch. Balty, « Un groupe dynastique d'époque tibérienne à Avignon. Notes sur l'iconographie de Drusus le jeune ", BACTHS, 26, 1998, p. 7-19.

8. L. Maurin, Saintes antique des origines à la fin $d u$ VI $I^{e}$ siècle apr. J.-C, Saintes, 1978. Voir aussi Bedon, 1999, p. 46-47 : les Santones y auraient installés leur chef-lieu après la conquête césarienne, à une vingtaine de kilomètres d'un oppidum antérieur. On peut situer la fondation de la ville vers 20 av. J.-C.

9. Cette prédominance des membres de la famille julio-claudienne est un phénomène commun à l'ensemble des grandes agglomérations gauloises.

10. Maurin, 1994, p.84.

11. Cf G. Gualandi, «L'apparato figurativo negli archi augustei », dans Studi sull'Arco Onorario Romano, Rome, 1979, p. 93-137 ; S. De Maria, Gli archi onorari di Roma e dell'Italia

Romana, Rome, 1988. Pour une liste de ces groupes, voir en particulier F. S. Kleiner, «The arch of Galba at Tarragona and Dynastic Portraiture on Roman arches », MM, 30, 1989, p. 239-252 et pl. 18.

12. J.-Ch. Balty, «Groupes statuaires impériaux et privés de l'époque julio-claudienne », dans Ritratto ufficiale e ritratto privato (Quaderni de La Ricerca scientifica, 116), Rome, 1988, p. 31-45.

13. Un arc a été élevé en 16 apr. J.-C. sur la Via Sacra qui honorait conjointement Tibère et Germanicus ; cf. F. S. Kleiner, art. cit., p. 246 : « statues of both emperor and adoptive son must have been exhibited on the attic $»$.

14. Hurlet, 1996, p. 527-531.

15. Saint-Germain-en-Laye, Musée des Antiquités Nationales, inv. $\mathrm{n}^{\circ} 12565 . \mathrm{H}=30 \mathrm{~cm}$.

16. CIL, XI, 1421.

17. Maurin, 1978, p. 79, note 56.

18. M. Roehmer, Der Bogen als Staatsmonument. Zur politischen Bedeutung der römischen Ehrenbogen des I. Jhs n. Chr., Munich, 1997, p. 158.

19. Maurin, 1978, p.107.

20. Texte $C$ (aius) Iuli[us], C(aii) Iuli(i) C[a]tuaneuni(i) f(ilius), Rufus, C(aii) Iuli(i) Agedomo[patis] nepos, Epotsoviridi(i) pron(epos), V[olt(inia)], [sacerdos Romae et Aug]usti [ad a]ram qu[a]e est ad Confluent $[e] m$, praefectus [fab]rum, $d(e)[s(u a) p(e c u n i a) f(e c i t)]$. (Caius Iulius Rufus, fils de Caius Iulius Catuaneunius, petit-fils de Caius Iulius Agedomopas, arrière-petit-fils d'Epotsoviridius, inscrit dans la tribu Voltinia, prêtre de Rome et d'Auguste à l'autel qui se trouve au Confluent, préfet des ouvriers, a élevé (cet $\operatorname{arc)}$ à ses frais). 
21. F. S. Kleiner, art. cit., p. 249 : « Private arches in honor of non-imperial persons are rare during the Empire. Before Galba three are attested that had multiple portraits, on the attic and/or in niches on the facades, honoring the families of those who paid for the monuments. "

22. Ti(berio) Caesar[i Diui Aug(usti) f(ilio) Diui Iuli nep(oti) Aug(usto)]/pontif(ici) max(imo), [co (n)s(uli) III], imp(eratori) VIII, [tri]b(unicia) pot(estate) [XXI]. Dr[us]o Caesari [Tib(erii) Aug(usti)] $f($ ilio)/[Divi Augusti] nep(oti), Divi Iuli/pronep(oti), co(n)s(uli)], pontifici, auguri. (À Tibère César Auguste, fils du divin Auguste, petit-fils du divin Jules, grand pontife, consul pour la troisième fois, salué empereur pour la huitième fois, en sa 21.(?) puissance tribunicienne.

À Drusus César, fils de Tibère Auguste, petit-fils d'Auguste, arrière petit-fils du divin Jules, consul, pontife, augure).

23. Germanico [Caesa]r[i] Ti(berii) Aug(usti) f(ilio)/Divi Aug(usti) nep(oti) Divi Iuli pronep(oti) aug (uri)/flam(ini) aug(ustali) co(n)s(uli) II imp(eratori) II. (À Germanicus César, fils de Tibère Auguste, petit-fils du divin Auguste, arrière-petit-fils du divin Jules, augure, flamine augustal, consul pour la deuxième fois, salué empereur pour la deuxième fois).

24. P. Grimal, « Deux inscriptions de Saintes », REA, 49, 1947, p. 134 : « il ne peut, en aucun cas, être postérieur à la mort de Germanicus ».

25. Id., art. cit., p. 135.

26. La Tabula Siarensis fait d'ores et déjà l'objet d'une bibliographie considérable ; on doit l'editio princeps à J. Gonzalez, in ZPE, 55, 1984 et J. Gonzalez et F. Fernandez, in Iura, 1981 [1984], p. 1-36; deux colloques se sont tenus à Séville : Estudios sobre la Tabula Siarensis, ed. J. Gonzalez, J. Arce, Madrid, 1988 ; une nouvelle édition a été donnée par J. Gonzalez dans Bronces juridicos romanos de Andalucia, Séville, 1990, p. 153-163 ; l'édition la plus commode est toutefois celle de l'Année Épigraphique, 1991, 20-22, p. 13-22, qui comprend, outre une traduction française, une synthèse sur l'ensemble des recherches les plus récentes.

27. Y figurent également Drusus l'Ancien, Antonia Minor, Agrippine l'Ancienne, Livilla, Claude, Néron César, Drusus César, Caligula, Agrippine la Jeune, Iulia Drusilla et Iulia Livilla ; pour un tableau commode des séries de dédicaces, voir Hurlet, 1996, p. 601.

28. Cf. Tacite, Ann., II, 82, 2.

29. Tab. Siar., frgt. 1, 1. 23-24 : «...sive qui] alius aptior locus Ti(berio) Caesari Aug(usto) principi nostr [o videretur in iis regionibus quarum] in curam et tutelam Germanico Caesari ex auctori[tate senatus mandasset] »; la traduction est tirée de l'Année Épigraphique, 1991, nº 20, p. 15.

30. Cf. Tab. Siar., frgt. 1, 1. 15 : « ordinato statu Galliarum ».

31. Pour une datation de l'arc postérieure à la mort de Germanicus, voir CIL, XIII, 1036 p. 137 ; A. Grenier, Manuel d'Archéologie gallo-romaine, I, Paris, 1931, p. 568 rem. 2. Tous ces auteurs s'appuient sur le passage de Tacite, Ann., II, 83, 2, mentionnant l'ampleur des honneurs funéraires rendus à Germanicus : « statuarum locorumue in quis coleretur haud facile quis numerum inierit ».

32. « Afin de perpétuer la mémoire de Germanicus César, qui n'aurait jamais dû mourir » : Tab. Siar., fragment I, 1. 1.

33. L. Maurin, 1994, p. 84.

34. P. Gros, « Une hypothèse sur l'arc d'Orange », Gallia, 44, 1986, p. 191-201.

35. Le monument, commencé vers 21 apr. J.-C., aurait été in fine dédié à Tibère entre juin 26 et juillet 27.

36. Cf. W. D. Lebek, « Ehrenbogen und Prinzentod : 9 v. Chr.- 23 n. Chr. », ZPE, 86, 1991, p. 68.

37. P. Grimal, « Deux inscriptions de Saintes », REA, 49, 1947, p. 135 sqq. 
38. J.-Ch. Balty et D. Cazes, Portraits impériaux de Béziers. Le groupe statuaire du forum, Toulouse, 1995, $n^{\circ} 5$ p. 72-76 (Tibère), $n^{\circ}$ 6, p. $79-85$ (Germanicus) et $n^{\circ}$ 7, p. 87-93 (Drusus le Jeune).

39. Le groupe d'Avignon, constitué des effigies de Tibère et Drusus le jeune, témoigne d'un moment ultérieur de l'histoire de la domus impériale : celui où, après la mort de Germanicus, Drusus, devenant le seul successeur possible, se voit décerner la puissance tribunicienne.

40. F. S. Kleiner, art. cit., p. 246, fait à ce propos preuve d'une prudence extrême, à juste titre : il mentionne en effet que l'arc de Saintes a été érigé « around A. D. 19 ».

41. Cf., sur ce point, les remarques de L. Maurin, 1978, p. 80-81 et de P. Gros, art. cit., note p. 196-197.

42. En Gaule ont été découverts dix statues ou bases de statues de Tibère (voir, pour la Narbonnaise, J. Gascou et D. Terrer, « La présence de Tibère en Narbonnaise : les portraits et les inscriptions ", RAN, 29, 1996, p. 31-68) ; quant à Drusus II, il est de loin le prince de la famille julio-claudienne le plus représenté, avec pas moins de huit témoignages (il est honoré à Nîmes, Ruscino, Castelculier, Béziers, Saintes, Avignon, Vienne et Saint-Maurice). 43. Ti(berio) Clau[dio]/[Cae] sari A[ug(usto)]/[Ger]manic(o), po[nt(ifici)]/max(imo) tribun(icia) pote[st(ate)]/VIIII, imp(eratori) XV, co(n)s(uli) II[II]/censori p(atri) [p(atriae)]/[C(aius) I] ulius, C (aii) f(ilius) Volt(inia), V[ictor]. (À Tibère Claude César Auguste Germanicus, grand pontife, revêtu de la puissance tribunicienne pour la neuvième fois, salué empereur pour la quinzième fois, consul pour la quatrième fois, censeur, père de la patrie, Caius Iulius Victor, fils de Caius, de la tribu Voltinia).

44. CIL, XIII, 1042 à 1045 ; Maurin, 1994, nº 18 p. 124-135.

45. P. Gros a amplement montré, au cours d'études consacrées à plusieurs villes de Gaule Narbonnaise (« L'Augusteum de Nîmes », RAN, XVII, 1984, p. 124-134 ; « Un programme augustéen : le centre monumental de la colonie d'Arles », JdI, 102, 1987, p. 339-363 ; " Théâtre et culte impérial en Gaule Narbonnaise et dans la péninsule ibérique », dans Stadtbild und Ideologie. Die Monumentalisierung hispanischer Städte zwischen Republik und Kaiserzeit (Madrid, 1987), Munich, 1990, p. 381-390) les modalités de ce qu'il appelle une « annexion de l'espace public » par l'image impériale d'un bout à l'autre d'une ville ; le cas de Saintes est à cet égard tout à fait exemplaire et ici s'ajoute encore l'annexion de cette image par une famille de notables locaux.

46. Wuilleumier, 1963, $\mathrm{n}^{\circ} 217$.

47. C'est peut-être la raison pour laquelle les titulatures impériales et donc les statues sont tournées du côté de la ville, sur la face occidentale de l'arc, ce qui pourrait surprendre sur un arc routier.

48. M. Roehmer, op. cit., p. 158 : « Der Bogen stand auf einer auffälligen, wichtigen Stelle. Jeder Reisende aus dem Norden und Osten mußte ihn vor seinem Eintritt in die Stadt passieren. »

49. W. Seston, «Les donateurs de l'amphithéâtre des Trois Gaules », dans Hommages à A. Grenier, Coll. Latomus, 58, 1962, p. 1414.

50. M. Roehmer, op. cit., p. 159 : « Der hauptsächliche Grund für den Bau dieses Monuments ist somit die Selbstdarstellung des C. Iulius Rufus ».

51. Hurlet, 1996, p. 468.

52. Cf M. Navarro Caballero, « Les dépenses publiques des notables des cités en Hispania Citerior sous le Haut-Empire », REA, 99, 1997, p. 136, qui présente une excellente analyse du phénomène : «L'analyse des éléments architecturaux et ornementaux qui concouraient au décor urbain révèle la signification idéologique du choix des notables qui 
les ont payés, stimulés par le pouvoir central : la volonté de coller au modèle romain, qui supposait leur intégration et permettait leur promotion ».

53. P. Zanker, « Nouvelles orientations de la recherche en iconographie. Commanditaires et spectateurs », RA, 1994, p. 281-293.

\section{RÉSUMÉS}

La ville de Mediolanum Santonum, capitale de l'Aquitaine augustéenne, a livré un ensemble important d'hommages statuaires et épigraphiques à la domus impériale julio-claudienne. Considérés jusqu'à présent comme des témoignages isolés, ils font en réalité partie d'un même programme idéologique qui fut orchestré, entre le règne de Tibère et celui de Claude, par une même famille de notables locaux exerçant des charges en relation avec le culte impérial. Instruments de propagande dynastique et d'annexion de l'espace public par l'image impériale, ces statues furent placées en un agencement scénographique en des points stratégiques de la ville au sein de groupes dont la composition a suivi les vicissitudes de la politique successorale de ces empereurs ; élevées à des tournants importants de l'histoire de la dynastie julio-claudienne, elles étaient donc destinées à la fois à susciter et à exprimer un consensus civique vis-à-vis du régime impérial, par l'intermédiaire d'un évergétisme municipal qui prenait en charge le financement et la mise en place de ces témoignages de loyalisme*.

Statues' life

Life of a statuary Julio-Claudian group in Mediolanum Santonum

The city of Mediolanum Santonum, capital of the Augustan Aquitaine, left us an important set of statuary and epigraphic tributes to the imperial Julio-Claudian domus. So far, they have been considered as isolated testimonies ; through, they actually belong to the same ideological programme, which was orchestrated, between the reign of Tiberius and the one of Claudius, by the same family of local worthies assuming charges linked with the imperial cult. Instruments of the dynastic propaganda and of the annexation of the public framework by the imperial image, these statues were set in a scenographic organization in strategic points of the city, among groups whose composition followed the vicissitudes of these emperor's policy of succession ; erected at important turning points of the Julio-Claudian dynasty, they were therefore meant both to arouse and to express a civic consensus towards the imperial regime, through the medium of a municipal evergetism, which was taking charge of the financial backing and of the setting of these testimonies of loyalism.

\section{AUTEUR}

\section{EMMANUELLE ROSSO}

Emmanuelle ROSSO travaille depuis 1996 sous la direction de J.-Ch. Balty (Université de Paris IV) sur la sculpture officielle romaine, plus précisément sur les portraits impériaux. Après avoir étudié, en maîtrise et en DEA, l'image de l'empereur dans les provinces gauloises, elle rédige une thèse de doctorat sur la propagande impériale et l'art officiel sous les Flaviens. Rattachée à l'Institut de Recherche sur l'Architecture Antique et au 
Centre Camille Jullian à Aix-en-Provence, elle participe à des fouilles et à des programmes de recherche dans le Sud de la France (Orange, Narbonne). Elle est moniteur de latin à l'Université de Provence. Rosso_emma(at)yahoo.fr 